\title{
CALIBRAÇÃO DE UM SISTEMA DE CHUVA SIMULADA POR DIÂMETRO DA GOTA PELO MÉTODO DA FARINHA E COEFICENTE DE UNIFORMIDADE
}

\author{
*Cicero Gomes"; Márcio Aurélio Lins dos Santos²; Silvania Farias Lima33; Igor Gledson de Oliveira Santos4; Tereza \\ Neuma Araújo de Carvalho ${ }^{5}$; Valdevan Rosendo do Santos ${ }^{6}$; Elvis da Silva Alves ${ }^{7}$
}

\footnotetext{
1*Prof. Doutor, Curso de Agronomia, Programa de Pós Graduação em Agricultura e Ambiente - UFAL, Arapiraca, AL. Av. Manoel Severino Barbosa, CEP 57309-005, Arapiraca, AL.fone (82) 99175-5434, e-mail: cgomes@arapiraca.ufal.br.

2Prof. Doutor, Curso de Agronomia, Programa de Pós Graduação em Agricultura e Ambiente - UFAL, Arapiraca, AL. e-mail: mal.santo@ gamil.com

3ZZotecnista, formada pela Universidade Federal de Alagoas - Campus Arapiraca. E-mail: silvaniaadmzoo@gmail.com.

${ }^{4}$ Mestrando, Agricultura e Ambiente, Universidade Federal de Alagoas, Av. Manoel Severino Barbosa, CEP 57309-005, Arapiraca, AL. E-mail: igor.gledson@gmail.com

5Engenheira Agrônoma, Campus Arapiraca, UFAL, Arapiraca, AL. e-mail: terezaufal@gmail.com.

6Prof. Doutor, Curso de Agronomia, Programa de Pós Graduação em Agricultura e Ambiente - UFAL, Arapiraca, AL. e-mail: valdevan@ yahoo.com.br

7Doutorando em Engenharia Agrícola, Universidade Federal de Viçosa - MG. E-mail: elvistv@gmail.com
}

RESUMO: A erosão do solo é considerada como a principal e mais generalizada forma de degradação do solo, consiste na remoção ou destacamento da camada superficial do solo pela água ou vento. 0 objetivo desta pesquisa foi calibrar o sistema de chuva simulada, através da determinação da uniformidade de distribuição, velocidade terminal e diâmetro de gota de chuva pelo método da farinha. A pesquisa foi realizada na Universidade Federal de Alagoas - Campus Arapiraca, utilizando simulador de chuva de acordo as especificações de Meyer; Harmon (1979). Na determinação do diâmetro da gota foi utilizado o método da farinha, descrito por Oliveira (1992). Os resultados para o diâmetro médio da gota de chuva apresentaram um comportamento muito semelhante, com pouca variação. As velocidades terminais das gotas de chuva nesta pesquisa não apresentaram grande variação em função dos intervalos de tempo de amostragem, com valores 6,65 a 3,64 m.s.' 0 coeficiente de uniformidade de Christiansen (CUC) obtidos foi em média acima de $75 \%$. 0 equipamento atende aos requisitos técnicos, que o capacita sua utilização como ferramenta de pesquisa nos processos erosivos.

PALAVRAS CHAVE: erosão, simulador de chuva, método de Christiansen.

\section{CALIBRATION OF A RAIN SYSTEM SIMULATED BY DROP DIAMETER BY THE FLOUR METHOD AND UNIFORMITY COEFFICENT}

\begin{abstract}
Soil erosion is considered the main and most widespread form of soil degradation, consisting of the removal or detachment of the topsoil layer by water or wind. The objective of this research was to calibrate the simulated rain system, by determining the uniformity of distribution, terminal speed and raindrop diameter by the flour method. The research was carried out at the Federal University of Alagoas - Campus Arapiraca, using a rain simulator according to the specifications of Meyer; Harmon (1979). In determining the droplet diameter, the flour method, described by Oliveira (1992), was used. The results for the average diameter of the raindrop showed a very similar behavior, with little variation. The terminal velocities of the raindrops in this research did not vary much as a function of the sampling time intervals, with values 6.65 to $3.64 \mathrm{~m}^{-1} \mathrm{~s}^{-1}$. The Christiansen uniformity coefficient (CUC) obtained was, on average, above $75 \%$. The equipment meets the technical requirements, which enable it to be used as a research tool in erosive processes.
\end{abstract}

KEY WORDS: erosion, rain simulator, method of Christiansen. 


\section{INTRODUÇÃO}

A erosão do solo é considerada como a principal e mais generalizada forma de degradação do solo, consiste na remoção ou destacamento da camada superficial do solo pela água ou vento, reduzindo os níveis de matéria orgânica e de nutrientes, com consequente diminuição da sua produtividade (Martins; Fernandes, 2017).

A água da chuva exerce ação erosiva sobre o solo pelo impacto das gotas, que caem com velocidade e energia variáveis, dependendo do seu diâmetro, e pelo escoamento da enxurrada, a qual tem sua velocidade e volume variando segundo a declividade do terreno, tamanho de declive e capacidade de infiltração de água no solo (Bertoni; Lombardi Neto, 2018).

A caracterização do padrão hidrológico das chuvas naturais para estudo de processos erosivos, requer vários anos de medidas, devido sua natureza irregular (Silva et al., 2015). Ainda de acordo com Silva et al. (2015b), uma forma de obtenção das características das chuvas, em intervalos mais curtos e com maior controle, se faz uso de precipitações artificiais, produzidas por equipamentos, denominados simuladores de chuva.

0 impacto das gotas de chuva na superfície do solo tem efeito importante no processo erosivo, pois provoca o destacamento e o salpicamento das partículas, disponibilizando-as para 0 arraste durante o escoamento superficial (Carvalho et al., 2012). As perdas de solo causadas pela erosão hídrica reduzem a espessura do solo, diminuindo a capacidade de retenção e redistribuição da água no perfil gerando, como consequência, maiores escoamentos superficial e, por vezes, maiores taxas de erosão do solo (Santos et al., 2010).

Os simuladores de chuva são ferramentas de pesquisa desenvolvidas para aplicar água de forma similar às chuvas naturais (Silva et al., 2015c). Os simuladores de chuva foram projetados e calibrados para produzir chuvas artificiais, que apresentem as características, entre elas gotas com diâmetro médios das chuvas e energia cinética e promovam uma distribuição uniforme da precipitação sobre a parcela experimental em estudo. Para desempenhar sua função de produzir chuvas mais próximas das naturais, estes equipamentos precisam serem calibrados através das características do diâmetro médio das gotas de chuva, distribuição uniforme e intensidade de chuva (Meyer; Harmon, 1979).

O objetivo desta pesquisa foi calibrar o sistema de chuva simulada, através da determinação da uniformidade de distribuição, velocidade terminal e diâmetro de gota de chuva pelo método da farinha de trigo nas condições de laboratório.

\section{MATERIAL E MÉTODOS}

0 experimento foi conduzido na Universidade Federal de Alagoas (UFAL) - Campus de Arapiraca, em ambiente protegido.

O simulador de chuva em estudo foi construído de acordo as especificações proposta por Meyer; Harmon (1979b), sendo equipado com um bico aspersor tipo Veejet 80-150 com diâmetro interno de $12,7 \mathrm{~mm}$, reproduz uma distribuição de tamanhos de gotas e níveis de energia cinética próximas as das chuvas naturais, que é de $0,294 \mathrm{MJ} \mathrm{ha}^{-1} \cdot \mathrm{mm}^{-1}$ e de $17,64 \mathrm{MJ} \mathrm{ha}^{-1}$.

Na determinação do diâmetro da gota foi utilizado o método da farinha, descrito por Oliveira (1991). As gotas, ao colidirem com a farinha formaram pequenos grânulos, esses foram secados ao ar por $24 \mathrm{~h}$, separados, utilizando peneiras de 0,25; 0,5; 1,0 e 2,0 mm, depois secos em estufa por $24 \mathrm{~h}\left(105^{\circ} \mathrm{C}\right)$ e, posteriormente, pesados em balança com precisão de $0,001 \mathrm{~g}$, obtendo-se a massa média dos grânulos. 0 diâmetro da gota foi determinada conforme a Equação (1):

$$
\mathrm{D}=\sqrt[3]{\frac{6 \mathrm{~m}}{\pi \rho}}
$$

em que: $D$ é diâmetro da gota $(\mathrm{mm}) ; \mathrm{m}$ é a massa média da gota $(\mathrm{mg})$ e p é a massa específica da água, em $\mathrm{mg} / \mathrm{mm}^{3}$.

A velocidade terminal é a máxima velocidade atingida pelas gotas precipitadas. Essa foi conforme a Equação (2):

$$
V T=\sqrt{\frac{9,81}{0.4671 d^{-0.9659}}}
$$

em que: VT é a velocidade terminal da gota $\left(\mathrm{m} . \mathrm{s}^{-1}\right)$ e d é o diâmetro da gota (mm).

0 volume de cada gota foi calculado de acordo com a Equação (3): 


$$
\mathrm{V}=\frac{\pi \mathrm{D}^{3}}{6}
$$

em que: $V$ é o volume da gota d'água $\left(\mathrm{mm}^{3}\right)$ e $D$ é 0 diâmetro da gota $(\mathrm{mm})$.

Para a determinação da intensidade de precipitação foram utilizados 25 copos coletores, distribuídos numa área de 0,6 m2, com tempo de coleta de 3 minutos. A Equação (4) utilizada foi conforme Ribeiro (2006a):

$$
I=\left[\frac{V / S}{t}\right] \cdot 60
$$

em que: I é a intensidade de precipitação $\left(\mathrm{mm} \cdot \mathrm{h}^{-1}\right)$; V é o volume de água coletado (L); S é a área da seção de coleta; e t é o tempo de teste.

O coeficiente de uniformidade de Christiansen (CUC) foi obtido através da seguinte Equação (5):

$$
\operatorname{CUC}=100\left(1-\sum_{i=1}^{n}|X i-X| / n X\right)
$$

em que: Xi é a lâmina precipitada em cada pote; X é a lâmina média precipitada; n é o número de potes.

\section{RESULTADOS E DISCUSSÃO}

Na Tabela 1, estão apresentados os resultados das determinações dos diâmetros médios de gotas e velocidade terminal em funções dos intervalos de amostragens e do diâmetro das peneiras em teste de chuva simulada.

Os diâmetros de gotas (Tabela 1) em função dos intervalos de amostragem apresentaram um comportamento muito semelhante, com pouca variação. Estes resultados ficaram abaixo dos obtidos por Ribeiro et al. (2006), que obteve um valor de 2,45 $\mathrm{mm}$, avaliando um minissimulador de chuva em Lavras $-M G$.

\begin{tabular}{|c|c|c|c|c|c|}
\hline Intervalo de tempo & Peneiras & $\begin{array}{l}\text { Massa média dos } \\
\text { grânulos }\end{array}$ & Diâmetro da gota & $\begin{array}{c}\text { Volume da gota } \\
\text { d'água }\end{array}$ & Velocidade terminal \\
\hline Min & $\mathrm{mm}$ & grama & $\mathrm{mm}$ & $\mathrm{mm}^{3}$ & $\mathrm{~m} \mathrm{~s}^{-1}$ \\
\hline 5 & 2,00 & 3,40 & 1,87 & 3,41 & 5,25 \\
\hline 5 & 1,00 & 1,20 & 1,32 & 1,20 & 4,13 \\
\hline 5 & 0,50 & 0,28 & 0,81 & 0,28 & 3,64 \\
\hline 5 & 0,25 & 0,13 & 0,63 & 0,13 & 6,50 \\
\hline 10 & 2,00 & 4,40 & 2,03 & 4,42 & 5,27 \\
\hline 10 & 1,00 & 1,23 & 1,33 & 1,23 & 3,85 \\
\hline 10 & 0,50 & 0,18 & 0,70 & 0,18 & 3,6 \\
\hline 10 & 0,25 & 0,12 & 0,61 & 0,12 & 6,37 \\
\hline 15 & 2,00 & 3,87 & 1,95 & 3,88 & 5,43 \\
\hline 15 & 1,00 & 1,46 & 1,41 & 1,46 & 4,21 \\
\hline 15 & 0,50 & 0,31 & 0,84 & 0,31 & 3,60 \\
\hline 15 & 0,25 & 0,12 & 0,61 & 0,12 & 6,65 \\
\hline 20 & 2,00 & 5,02 & 2,13 & 5,04 & 5,45 \\
\hline 20 & 1,00 & 1,50 & 1,42 & 1,50 & 3,94 \\
\hline 20 & 0,50 & 0,21 & 0,74 & 0,21 & 3,64 \\
\hline 20 & 0,25 & 0,13 & 0,63 & 0,13 & 5,25 \\
\hline
\end{tabular}

Tabela 1. Médias dos diâmetros das gotas, velocidade terminal durante os intervalos de tempo de 5, 10, 15 e 20 minutos de precipitação da chuva simulada.

Silva et al. (2015) calibrando um sistema de simulador de chuva em função de três intervalos de amostragem, obteve resultados semelhantes, com valores que variam de 2,38 a 2,54 mm.

Na determinação do diâmetro médio de gotas de chuva, considera-se a gota como uma esfera perfeita (Ribeiro et al., 2006c. Este método vem sendo utilizado a mais de cem anos, inicialmente com eventos de chuva natural, a partir da década de 60 , com chuva simulada, com a determinação média de gotas até um diâmetro mínimo de 0,3 mm (Carvalho et al., 2012).

As velocidades terminais das gotas de chuva nesta pesquisa não apresentaram grande variação em função dos intervalos de tempo de amostragem, com 
valores 6,65 a 3,64 m.s.-1 (Tabela 1). Estes valores estão condizentes com os obtidos por Keller (1984), que afirmam que as velocidades terminais podem variar de 2 a 22 m.s. $^{-1}$.
Na Figura 2, observa-se a curva de velocidade terminar versus volume da gota de chuva, com características semelhantes as descritas por Meyer; Harmon, (1979), com diferentes intervalos de amostragem.

Figura 1. Velocidade terminal versus diâmetro de gota avaliadas pelo método da farinha para o teste de chuva simulada.

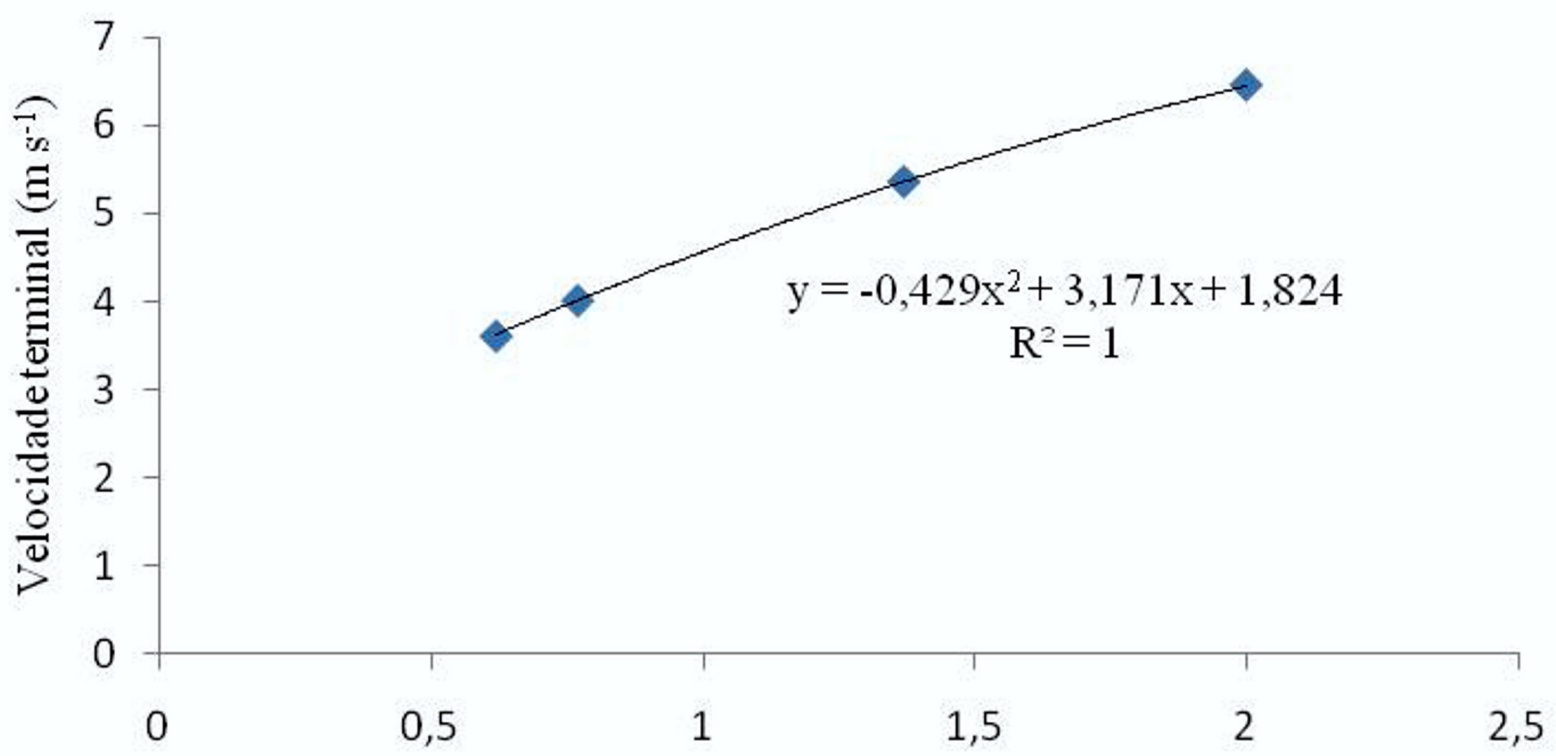

Diâmetro da gota (mm)

Figura 2. Velocidade terminal versus volume de gota avaliadas pelo método da farinha para o teste de chuva simulada.

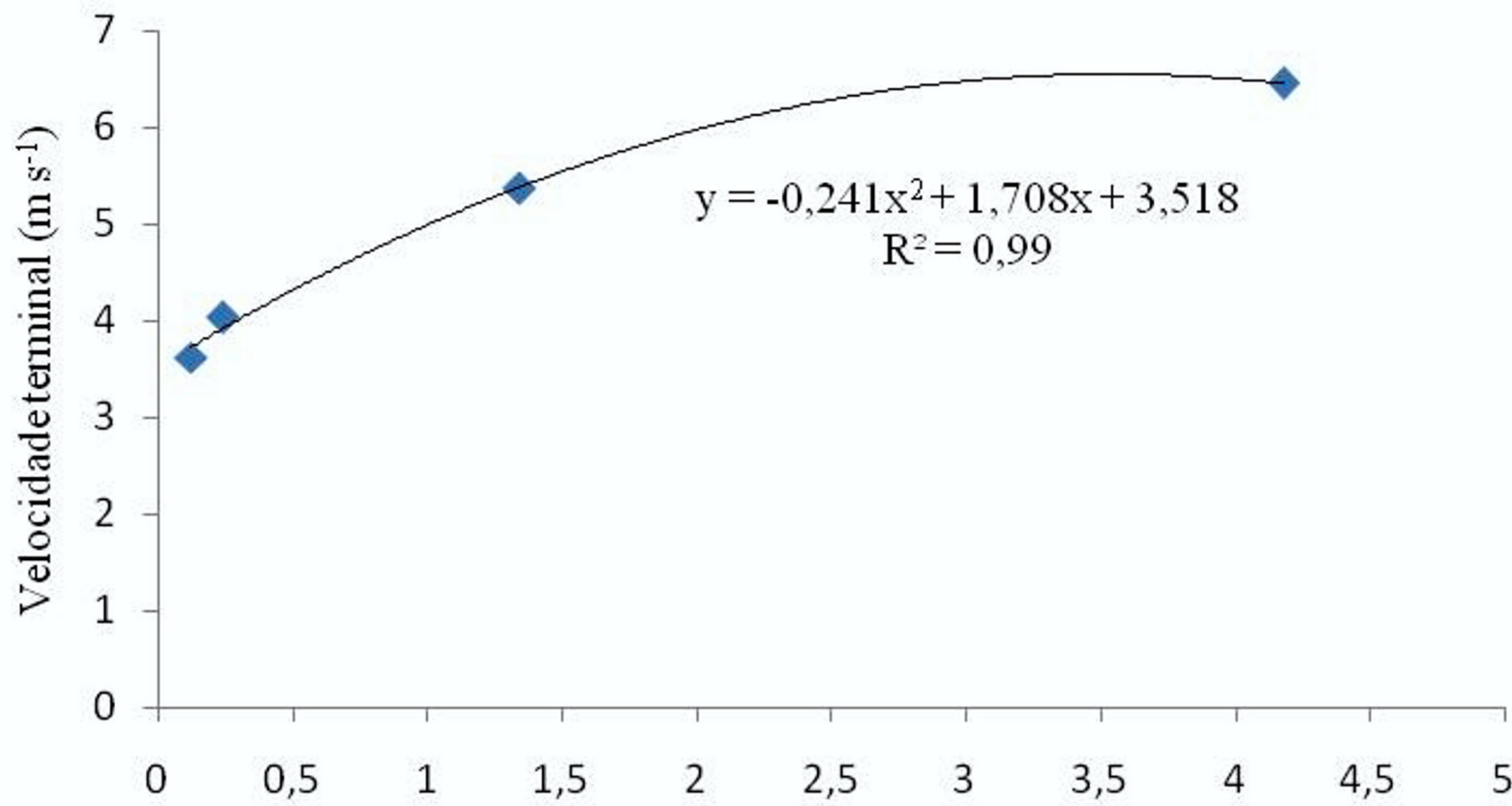

Volume da gota $\left(\mathrm{mm}^{3}\right)$ 
Os valores de gota de chuva obtidos apresentam uma ampla variação, com uma velocidade terminal acima de $3 \mathrm{~mm}$ e atinge uma velocidade de aproximadamente $6 \mathrm{~mm}$. Segundo Silva et al. (2015) no estudo do processo erosivo merece destaque a importância da avaliação do volume médio que as gotas atingem em relação a sua velocidade terminal, pois terão uma relação direta com a força de impacto sobre o solo, contribuindo para uma maior ou menor taxa de desagregação.

0 resultado da avaliação do coeficiente de uniformidade de Crhristiansen (CUC) apresentam um percentual de $82 \%$ a um intervalo de 5 minutos de teste (Tabela 2) para um equipamento simulador de chuva, utilizando uma pressão de serviço 41 kpa e utilizandose um bico 80-150.

Tabela 2 - Valores de coeficiente de uniformidade de Christiansen (CUC) e valores de intensidade de precipitação obtidos com um equipamento de chuva simulada.

\begin{tabular}{ccc}
\hline Intervalo de tempo $(\mathrm{min})$ & CUC $(\%)$ & Intensidade de precipitação $\left(\mathrm{mm} \cdot \mathrm{h}^{-1}\right)$ \\
\hline 5 & 82,95 & 19,9 \\
\hline 10 & 78,61 & 25,01 \\
\hline 15 & 78,65 & 25,16 \\
\hline 20 & 87,49 & 16,8 \\
\hline
\end{tabular}

Montebeller et al., (2001), avaliando as características hidráulicas, com diferentes intensidades de pressão e com bico diferentes, obteve na CUC de $80 \%$ em relação uniformidade, verifica-se que à medida que se aumenta a pressão de serviço do bico, existe uma sensível aumento no coeficiente da uniformidade.

Desta forma, o método da farinha permitiu avaliar o funcionamento do simulador de chuva, através das variáveis diâmetros médios das gotas de chuva simulada e velocidade terminal.

\section{REFERÊNCIAS BIBLIOGRÁFICAS}

Bertoni. J.; Lombardi, Neto, F. Conservação do Solo. São Paulo: Editora Ícone, 2018.

Carvalho, M.F.; Tarqui, J. L. Z.; Silva, V. S.; Lobo, B. R. de H. Avaliação do Funcionamento de um Simulador de Chuva Pelo Método da Farinha. Revista Brasileira de Recursos Hídricos, 2012, 17, 115-124.

Martins, C.; Fernandes, R. Processos de degradação do solo - medidas de prevenção. Dossier Técnico, Vida Rural. 2017.
Meyer,L.D. Rain fall simulators for for soil erosion research. In: LAL, R. (Ed). Soil Erosion Research Methods. Delray Beach: St. Lucie Press. 1994, p. 83103.

Ribeiro, B. T. Energia cinética de gotas de chuva simulada e energia ultra-sônica na desagregação de um Cambissolo. Dissertação. Lavras, MG, 2006.

Santos, G. G.; Griebeler, N. P.; Oliveira, L. F. C. de. Chuvas intensas relacionadas à erosão hídrica. Revista Brasileira de Engenharia Agrícola e Ambiental, 2010, 14, 2, 115-123.

Silva, J. J. Da.; Bezerra, S. A.; Ribeiro, P. R.; Barros, A. P. S. De M.; Silva, E. De L.; Pereira, R. A. Determinação das Médias dos Diâmetros das Gotas de um Simulador de Chuvas para servir de Ferramenta para Pesquisas Científicas Relacionadas à Erosão Hídrica Utilizado - Método da Farinha de Trigo. XXXV Congresso Brasileiro de Ciência d Solo, 2015. 